\title{
Function over form
}

\author{
Nature Climate Change is making changes to our article formats to streamline our content and more clearly denote \\ original research contributions.
}

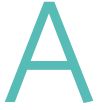
t Nature Climate Change, we aim to publish research from all areas of the natural and social sciences of relevance to climate change. Accommodating this breadth of research is not trivial, as different disciplines have different norms and styles for scientific communication. We strive to maintain a balance between consistency in the look and feel of Nature Climate Change papers and ensuring that they meet the needs of different research communities. As we approach our tenth anniversary next April, we are taking stock of our current set of available format options and making changes to reflect how the journal and research landscape have evolved.

Nature Climate Change followed the model set by Nature when it launched, offering both Letter and Article formats for primary research (we consider as primary research any paper that presents conclusions based on new data and/or analysis). Letters were intended for brief communication of important research results, whereas Articles offered additional space for work considered more substantial. In practice, however, the distinction became one of length alone, particularly as we began publishing more social science and interdisciplinary research; we were often asking authors to revise their Letter as an Article to allow for more space to accommodate the additional discussion or explanation requested by reviewers. Consequently, the different formatting specifications for Letters versus Articles (such as a bold introductory paragraph with references versus a traditional abstract with no references) is difficult to justify; these differences are superficial rather than substantive, and ultimately create unnecessary work for authors and editors alike. Thus, although we still encourage authors to be as succinct as possible in presenting their research, following the lead of the flagship journal (Nature 574, 453; 2019) and to be consistent with more recent additions to the Nature family, such as Nature Energy, Nature Sustainability and Nature Food, we are retiring the Letter format.

Although we no longer wish to distinguish primary research papers on the basis of arbitrary differences in length and form, we recognize that research can have different aims, and this should be more clearly highlighted to our readers. To that

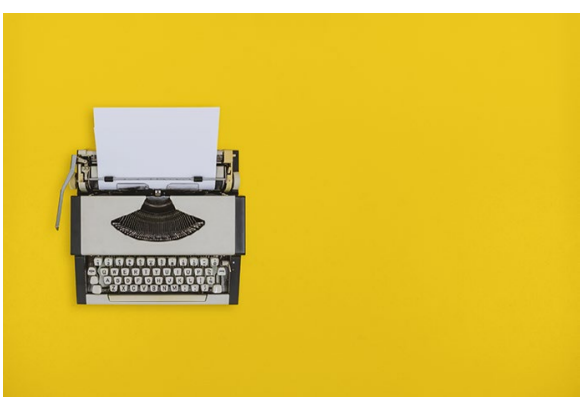

Credit: CONSTANTINE JOHNNY / MOMENT / GETTY

end, we are introducing Analyses, which have the same formatting as Articles and are held to the same editorial and technical standards, but represent a different kind of contribution: whereas Articles present a conceptual advance to the literature, the value of an Analysis lies in the execution of the analysis itself.

For instance, in our first Analysis published in this issue, Groom et al. find that updating the Dynamic Integrated ClimateEconomy (DICE) integrated assessment model makes previously unachievable temperature targets attainable. This does not necessarily advance understanding of the factors driving the economic feasibility of these targets, but demonstrating the impact of model modifications is valuable for the field given the centrality of the DICE model. Another example from our archives that we would now consider as an Analysis is a framing analysis of climate change editorials in Nature and Science (Nat. Clim. Change 8, 515-521; 2018). The specific findings in that study do not necessarily extend understanding of media coverage of climate change, but we felt the analysis itself would be of interest to our readership.

The final change is the introduction of Brief Communications. Historically, our Comment and Correspondence sections have included pieces that present original data and analysis (such as Peters et al. (Nat. Clim. Change 7, 848-850; 2017), Mitchell et al. (Nat. Clim. Change 8, 551-553; 2018) and Betts et al. (Nat. Clim. Change 6, 806-810; 2016)). However, this has led to a lack of clarity about whether such pieces were peer-reviewed (Comments and Correspondence are peer-reviewed at the editor's discretion) and to what standards, as well as uncertainty as to whether they should be considered as research for the purposes of tenure and promotion. Moreover, presenting new analysis in a Comment or Correspondence prevents authors from making use of new features such as extended data and source data, which allow for greater accessibility of supplementary figures and underlying data.

In light of these considerations, we are restricting our Comment and Correspondence sections to opinion pieces, and no longer accept Comments or Correspondence that develop arguments on the basis of new data and/or analysis. At the same time, the volume of data-heavy Comment and Correspondence submissions we receive suggests that there is a strong appetite for brief analytic pieces, particularly within the physical climate and climate modelling communities. Brief Communications will fill this gap. Like our other primary research formats, all Brief Communications will require a formal methods section that includes data and code availability statements to promote transparency and reproducibility, and they will always be peer-reviewed.

We hope these new formats will help us accommodate the majority of work we receive. But given the diversity of the research landscape we cover, we still anticipate encountering submissions that do not obviously fit the available options. We also know that it can be complicated for authors to navigate our different formats. Remember that we do not require initial submissions to be formatted in Nature Climate Change style, nor do we reject papers on the basis of submission as the 'wrong' format type; our editors guide authors to the format that best suits the contribution of the work either before review or once that contribution has been clarified based on reviewer feedback. Ultimately, our goal with these changes to article formats is not to complicate submission or evaluation procedures, but rather to ensure that all primary research is clearly denoted as such, and to better indicate the kind of contribution on offer to our readers.

Published online: 29 July 2020 https://doi.org/10.1038/s41558-020-0859-0 